greater confidence in the detected signal than in the nature of its source. Based on a single firing of an Aerobee rocket just over a year ago carrying a $17 \mathrm{~cm}$ telescope to a height of $170 \mathrm{~km}$, the result was an infrared flux in the range 0.4 to $1.3 \mathrm{~mm}$, nearly a hundred times that expected.

The two new papers in Physical Review Letters say that if the excess of far-infrared radiation is genuine then it neatly accounts for the gamma ray observations, through the action of high energy electrons on the far-infrared radiation in the inverse Compton effect. Shen also writes that the gamma ray maximum in the direction of the galactic centre may reflect the presence there of an intense infrared source, or alternatively that the galactic centre is a strong source of cosmic rays.

But neither the Indian team nor Shen at Purdue attempts to answer the question of the origin of the far-infrared radiation, which corresponds to a blackbody temperature of $8 \cdot 3^{\circ} \mathrm{K}$, embarrassingly larger than the well-known $3^{\circ} \mathrm{K}$ radiation which permeates space. Another way of looking at the measurements is that the radiation is a factor of 30 greater than the total radiation expected in a $3^{\circ} \mathrm{K}$ cosmic flux. Clearly the authors of the report are baffled-they say there are arguments against atmospheric, solar system and cosmic origins for their result. Nevertheless, it is gratifying that two recent problems in astronomy may now have been condensed into one.

\section{CRETACEOUS REVERSALS}

\section{Long Normal Period}

\section{from our Geomagnetism Correspondent}

Combrned palacomagnetic evidence from continental rocks and deep occan sediments, together with analyses of ocean magnetic anomalies, shows that during the Tertiary the geomagnetic field spent approximately equal periods in the normal and reversed states. But the average length of each period of constant polarity seems to vary with time. During the past ten million years, the average time between reversals has been about $0.22 \mathrm{~m} . \mathrm{y}$., a decrease from about $1 \cdot 0 \mathrm{~m} . \mathrm{y}$. for the older Tertiary. It is well known, too, that geological time includes at least one very long period of constant polarity - the normal Kiaman interval which covers the Permian and part of the Carboniferous.

Reversals in the Tertiary are now fairly well documented; and the Kiaman interval is conspicuous by its length. The pattern of reversals in other geological periods is less well known. New continental palaeomagnetic data for the Cretaceous, obtained by Helsley and Steiner (Earth and Planet. Sci. Lett., 5, 325; 1969), have been combined with all previous data for this period in an analysis which suggests that the roversal pattern in the Cretaceous was very different from that in the Tertiary. Eighty per cent of continental Cretaceous rocks are found to be magnetized normally, which means that the geomagnetic field was normal for 80 per cent of the Cretaceous epoch if palacomagnetic sampling is random in time. Furthermore, the reversed specimens discovered thus far are confined to the Lower Cretaceous and the upper part of the Upper Cretaceous, leaving a long normal period of at least $25 \mathrm{~m} . \mathrm{y}$. lying between about $80 \mathrm{~m} . \mathrm{y}$. and $105 \mathrm{~m} . \mathrm{y}$.

According to Helsley and Steiner, further evidence for the existence of this long normal period can be obtained from ocean magnetic anomalies. Heirtzler et al. (J. Geophys. Res., 73, 2199; 1968), using ocean magnetic surveys, have postulated a reversal pattern which, assuming a constant ocean floor spreading rate, extends backwards in time to about $80 \mathrm{~m} . \mathrm{y}$. Other surveys indicate a long normal interval of at least $20 \mathrm{~m} . \mathrm{y}$. (based on reasonable assumptions of spreading rate) extending backwards from Heirtzler's anomaly number 32 at $78 \mathrm{~m} . y$. Helsley and Steiner thus argue that this long interval correlates with their long normal Cretaceous period. If this is the case, then the ages assigned in this range by Heirtzler et al. are about $15 \mathrm{~m} . \mathrm{y}$. too great, though this is not too large an error bearing in mind the limitations on the constant spreading rate assumption. The important point is that the long normal period observed in continental rocks may also be detected in magnetic surveys in approximately the correct position and lasting about the same time.

The inherent danger in this type of analysis is the possibility that polarity epochs and events may yet remain undetected in continental rocks. Continental igneous rocks are not formed continuously; and thus a continuous time sequence may not be obtained by sampling, however thorough. For this reason the number of reversals observed within a given period may only be regarded as a minimum. Short, difficultto-resolve polarity events are still being discovered even within the comparatively well-established pattern of the past $3.5 \mathrm{~m} . \mathrm{y}$.

\section{SOLID STATE PHYSICS \\ Interacting Excitations}

\section{from a Correspondent}

WITH the aim of bringing together solid state theorists to discuss the theoretical methods available in different physical situations, the Institute of Physics and the Physical Society organized a conference on interactions among elementary excitations in solids and liquids at the University of Nottingham on March 25-27. The response to this experiment was disappointing: fifteen papers were presented, eight of which were invited, and there were fewer than fifty participants.

A highlight of the conference was the introductory talk by S. F. Edwards (University of Manchester), who emphasized the non-linearity of the equations satisfied by one-particle Green functions in many-body systems, and the limitations of perturbation theory. He illustrated the difficulties by discussing the simpler case of the one-electron theory of an alloy, or liquid metal. It was pointed out that, even when the poten. tial due to the disordered ions is very weak, there is always a strong-coupling regime at the bottom of the low-energy tail in the density of states curve. This is quite analogous to the critical region near a phase transition in the true many-body systom. Professor Edwards produced the remarkable result that in this strong-coupling regime the kinetic energy is not represented by $\mathrm{h}^{2} \mathrm{k}^{2} / 2 \mathrm{~m}$ but is proportional to $\mathrm{k}^{4 / 3}$. Nonlinearity was also emphasized by K. W. H. Stevens (University of Nottingham) in his discussion of strongly coupled spin-photon and spin-phonon systems. $\mathrm{He}$ described how a two-level spin system, under the influence of a travelling electromagnetic wave, cycles periodically between the two levels. Thus energy is 